\title{
空间动态可变材质的交互式全局光照明绘制
}

孙 金峑 ${ }^{+}$, 周 昆 ${ }^{2}$, 石教英

${ }^{1}$ (浙江大学 $\mathrm{CAD} / \mathrm{CG}$ 国家重点实验室, 浙江 杭州 310027)

${ }^{2}$ (微软亚洲研究院 网络图形组, 北京 100190)

\section{Interactive Global Illumination Rendering with Spatial-Variant Dynamic Materials}

\author{
SUN Xin ${ }^{1+}, \quad$ ZHOU Kun ${ }^{2}, \quad$ SHI Jiao-Ying ${ }^{1}$ \\ ${ }^{1}$ (State Key Laboratory of CAD\&CG, Zhejiang University, Hangzhou 310027, China) \\ ${ }^{2}$ (Internet Graphics Group, Microsoft Research Asia, Beijing 100190, China) \\ + Corresponding author: E-mail: sunxin@cad.zju.edu.cn
}

Sun X, Zhou K, Shi JY. Interactive global illumination rendering with spatial-variant dynamic materials. Journal of Software, 2008,19(7):1783-1793. http://www.jos.org.cn/1000-9825/19/1783.htm

\begin{abstract}
This paper proposes a method of interactive global illumination rendering with spatial-variant dynamic materials under complex illumination. With the spatial-variant dynamic materials, the materials of the scene can be changed while rendering, and the changes to different parts of an object can be different. The non-linear relationship between materials and out-going radiance prevents users from changing the materials with most of existing interactive global illumination rendering algorithms. If different parts of an object are covered with different materials, the materials take much more complex effects on the out-going radiance. So there is still not an interactive global illumination rendering algorithm allow users to make different changes to different parts of an object. This paper approximates a region with spatial-variant dynamic materials by dividing it into numbers of sub-regions, the material in each sub-region is uniform and consistent. The radiance transferred in the scene may be reflected by different sub-regions successively, and the paper divides the out-going radiance according to different sequences of the reflection sub-regions. This paper also represents all materials with a linear basis, and applied the basis to all sub-regions to get all different distributions of the material basis. This paper precomputes all parts of radiance of all material basis distributions. In rendering process, it uses the materials' coefficients of basis to combine corresponding precomputed data to achieve the global illumination effects with interactive performance.
\end{abstract}

Key words: spatial-variant; global illumination; bidirectional reflectance distribution function (BRDF); path tracing; rendering equation; principle component analysis (PCA)

摘 要: 提出了一种空间动态可变材质的交互式全局光照明绘制算法.如果在绘制过程中允许用户对物体的材质 作修改, 并且对一个物体的不同部分的材质作不同的修改,则称为空间动态可变材质. 由于最终出射的辐射亮度和材 质呈非线性关系,因此现有许多交互式全局光照明算法不允许用户修改物体的材质.如果一个物体各部分的材质可 以不相同, 那么材质对最终的出射的辐射亮度的影响更为复杂, 目前没有任何交互式全局光照明绘制算法能够在绘

* Supported by the National Basic Research Program of China under Grant No.2002CB312105 (国家重点基础研究发展计划(973)) Received 2007-07-04; Accepted 2007-12-24 
制过程中对一个物体不同部分的材质作不同的修改.将一个空间动态可变材质区域划分成许多子区域来近似模拟， 每个子区域内部材质处处相同. 光在场景传播过程中可能先后被不同的子区域反射, 并以此将最终出射的辐射亮度 分为许多部分. 用一组基材质来线性表示所有的材质,这组基材质被赋予场景中的所有子区域,从而得到不同的基材 质的分布.预计算所有这些基材质分布下的各部分最终出射的辐射亮度.绘制时根据各子区域材质在基材质上的系 数组合相应的预计算数据, 就能交互式绘制全局光照明效果.

关键词: 空间动态;全局光照明;双向反射分布函数;路径跟踪;绘制方程;主元分析法 中图法分类号: TP391 文献标识码: A

全局光照明技术可以生成照片级真实感的图像. 根据Kajiya ${ }^{[1]}$ 提出的绘制方程来模拟光在虚拟场景中的传 播行为, 可以得到现实世界中的各种光影效果, 包括光源与物体直接作用产生的直接光照明效果, 以及由于光能 在物体之间的相互反射产生的间接光照效果.最终产生的图像决定于光源、场景中物体的几何形状、相互位置 关系以及物体的表面材质.

其中,物体的材质是一个非常重要的属性, 它不但使物体在直接光照下表现出各种不同的质感, 而且它可以 改变光的传播路径从而影响到场景中其他区域的间接光照明效果. 如果在进行绘制时, 考虑到物体之间的遮挡 以及间接光照的效果,则称其为全局光照明绘制;反之,则称其为局部光照明绘制.

如果在用户可以通过交互修改虚拟场景中物体的材质,并且能以实时或者交互式的性能得到全局光照明 的绘制效果,则称其为可变材质.光在场景中传播, 可能经过多次反射, 每次反射都受到材质的作用, 因此材质和 绘制结果呈非线性关系. 而现有大多数实时或交互式全局光照明绘制算法都要利用出射亮度和入射亮度的线 性关系, 还需要假定材质固定不变,因此这些算法无法直接用于可变材质.一种简单的可变材质是空间静态可变 材质,即一个物体各个部分的材质完全相同,而且经过用户修改后也是如此, 目前的可变材质的实时或交互式全 局光照明绘制算法都是在这样的假设下实现的.如果一个物体各部分的材质可能不同,并且用户可以对不同部 分的材质作不同的修改,则称其为空间动态可变材质.与空间静态可变材质相比, 空间动态可变材质对光传播的 影响更加复杂,目前尚没有任何支持空间动态可变材质的交互式全局光照明绘制的算法.

现实世界中,大多数物体都被不同的材质覆盖不同的部分,因此空间动态可变材质相对于空间静态可辨材 质更为通用, 而且能够带来更加丰富的视觉效果. 如图 1 所示的 4 张图都是全局光照明的绘制效果:图 1(a)和 图 1(b)主要体现了不同颜色物体之间的渗色(color bleeding)效果, 图 1(c)和图 1(d)主要体现了高光材质的圆环 反射到平面上的焦散(caustics)效果. 图 1(a)和图 1(c)是空间静态可变材质,在同一个物体上的材质处处相同,图 1(b)和图 1(d) 是空间动态可变材质,一个物体上的不同部分被不同的材质覆盖. 可以明显看到空间动态可变材质 能够体现更为丰富的细节特征和高频的材质效果.

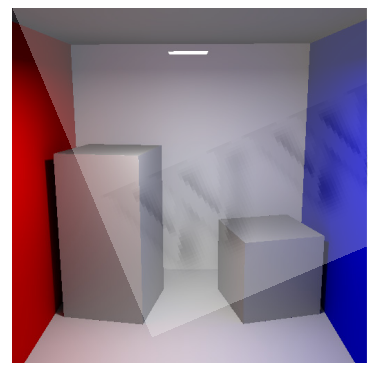

(a)

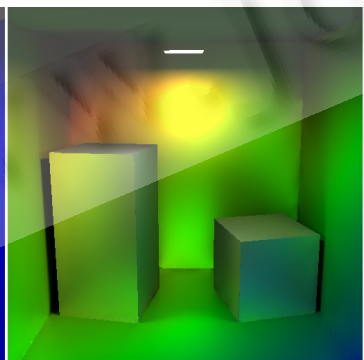

(b)

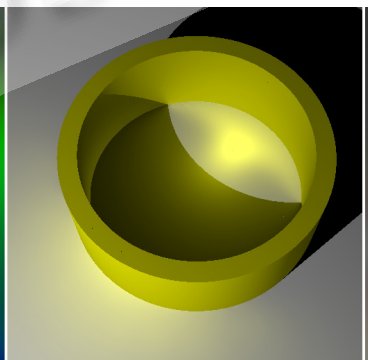

(c)

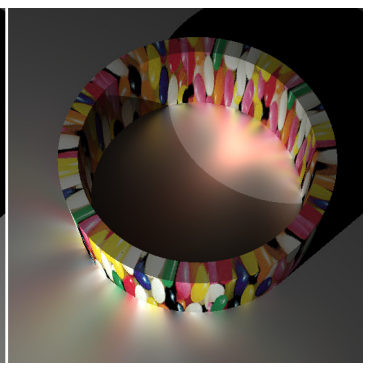

(d)

Fig.1 Global illumination with spatial-static materials and spatial-variant materials.

(a), (c) spatial-static materials; (b), (d) spatial-variant materials

图 1 空间静态材质和空间动态材质的全局光照明绘制

(a),(c)空间静态材质;(b)(d)空间动态材质 
在本文中,我们致力于为空间动态可变材质交互式生成全局光照明的绘制效果.具体描述为:采用与 Ben-Artzi ${ }^{[2]}$ 相同的设置, 在静态场景中采用复杂光源(本文采用的是面光源), 并且在空间动态材质变化时能够 实时地得到全局光照明的绘制效果. 我们采用Blinn-Phong ${ }^{[3]}$ 的双向反射分布函数 (bidirectional reflectance distribution function,简称BRDF) 描述物体的材质.

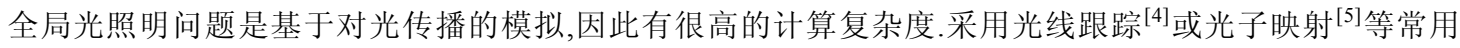
的离线全局光照明技术,在对场景所作的任何改变过程中,包括改变物体的材质,都需要重新绘制,在采用点光 源和方向光源以外的复杂光源时, 无法实时得到绘制结果.Sloan ${ }^{[6]}$ 提出的预计算辐射传输(precomputed radiance transfer,简称PRT)和周昆 ${ }^{[7]}$ 提出的阴影场(shadow fields)的方法,将光照或遮挡反射等因素的影响分解到线性基 上,将积分运算转化为基的系数的操作,实现了动态光源下的实时绘制. 但是,物体材质和全局光照明绘制效果 之间不存在这样的线性关系, 因此Ben-Artzi ${ }^{[2]}$ 只完成了直接光照下可变材质的实时绘制. 为了引入间接光照,孙 金金 ${ }^{[8,9}$ 将辐射强度根据传播路径进行分类, 从而建立起每一类辐射传输和各自相关材质的线性关系, 实现了空间 静态可变材质的全局光照明绘制. 当引入空间动态材质后, 将导致这种传输路径的分类的数量变得极其巨大,使 得无法进行实时绘制,这就是本文需要解决的关键问题.

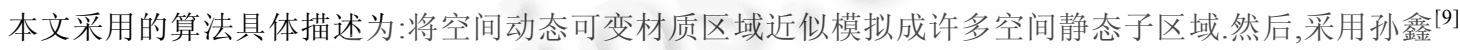
的方法进行预计算. 由于子区域数量多,这样得到的预计算结果数据量巨大,不能直接用于绘制. 为了减少数据 量,我们不考虑BRDF的高光部分的相互反射,但是我们计算漫反射之间以及慢反射和高光之间的相互作用; 类 似的假设普遍用于重光照技术中,比如Hasan ${ }^{[10]}$ 假设只考虑漫反射的反射作用,不同的是我们的假设中还保留 了从高光到漫反射的间接光照,所以我们能够实现焦散等高频的间接光照的绘制效果.我们发现,预计算结果具 有很大的相关性和稀疏性,因此我们采用PCA对不同光路的数据进行压缩,然后对得到的结果再使用Harr小波 进行压缩.这样,最后得到的结果有合理的数据量,能够用于交互式绘制.

综上所述,本文的创新主要有两点:一是采用划分子区域的方法来模拟空间动态可变材质; 二是利用计算数 据的相关性和稀疏性进行压缩,从而达到交互式绘制的性能.

本文的方法可以在场景建模时为空间动态材质的选择和编辑提供全局光照明的反馈效果,比如纹理贴图 的选择和编辑,而目前大多数工具只能提供局部光照明的反馈效果.

\section{1 相关工作}

\section{1 全局光照明及图像空间的重光照}

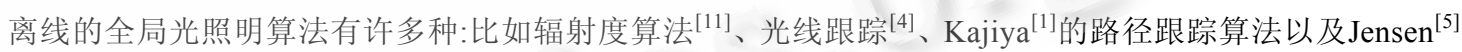
的光子映射(photon mapping)算法等,现有的其他离线全局光照明算法基本都是以上方法的扩展或优化.这些算 法一般无法用于实时绘制,尤其是在采用复杂光源和非镜面的高光材质时,绘制时间和空间上的代价非常巨大.

重光照技术一般有两种思路:把可选择的光照投影到基上以加速绘制, 比如Dorsey ${ }^{[12,13]}$ 和Dobashi ${ }^{[14]}$; 用图 像的像素存储几何信息, 比如 Saito ${ }^{[15]}$ 的几何帧缓存,之后几何帧缓存被扩展为深度帧缓存 ${ }^{[16,17]}$, 而 $\mathrm{Hasan}^{[10]}$ 将这 类重光照的方法提高到实时的绘制速度.

\section{2 预计算辐射传递}

利用光照和绘制效果的线性关系以及GPU(graphics processing unit)的优越性能,Sloan提出的PRT框架 ${ }^{[6,18]}$ 能够实时绘制固定场景在动态环境光下的低频的全局光照效果. $\mathrm{Ng}^{[19]}$ 通过引入Haar小波基, 得到了高频的光照 效果,但是对于非漫反射材质无法实时改变视点.对此, $\mathrm{Ng}^{[20]}$ 将绘制方程表述为一个三重积(triple product) 问题. 此外,对非漫反射的BRDF进行分解 ${ }^{[21,22]}$ 也是解决该问题的一种可行的策略. 周昆 ${ }^{[7]}$ 提出的阴影场实现了动态场 景和局部光源的直接光照的实时绘制.

\section{3 双向反射分布函数}

$\mathrm{BRDF}^{[23]}$ 是一种描述反射特性的材质模型. 有的BRDF模型关于反射点的法向中心对称,我们称其为各向同 


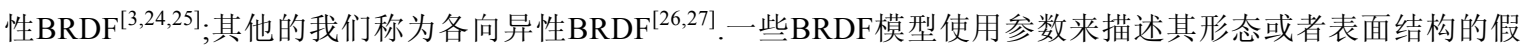
设,并有显式解析表达 ${ }^{[3,24-26]}$; 有一类BRDF, 直接从真实材质采集得到 ${ }^{[27]}$.

\section{4 双向反射分布函数的编辑}

解析形式BRDF的编辑可以通过修改其参数来实现 ${ }^{[9]}$, 也有软件支持用户订制的 $\mathrm{BRDF}^{[28]} \cdot \mathrm{McCool}^{[29]}$ 通过 分解BRDF加速绘制.Matusik ${ }^{[27]}$ 使用线性组合的方法从已有的BRDF生成新的BRDF.Ben-Artzi ${ }^{[2]}$ 分离出BRDF 的用户控制分量(user-controlled variable)提供给用户进行材质编辑.

\section{5 可变材质的实时绘制}

Ben-Artzi ${ }^{[2]}$ 通过抛弃间接光照, 得到绘制效果和材质的线性关系, 从而利用PRT框架实现可变材质的直接 光照的实时绘制. 孙金金 $[8$, 通过将光的传播路径进行分类,建立每一类的辐射传输和材质的线性关系, 实现空间 静态可变材质的全局光照明的实时绘制.

\section{2 算法概述}

若物体没有自发光, 且场景中不存在透明或半透明材质,则根据绘制方程 ${ }^{[1]}$ 可将可全局光照明问题描述为

$$
\begin{gathered}
L\left(x, x^{\prime}\right)=\int_{\Omega / 2} L\left(x^{\prime}, x^{\prime \prime}\right) \rho\left(x, x^{\prime}, x^{\prime \prime}\right) G\left(x, x^{\prime}, x^{\prime \prime}\right) \mathrm{d} x^{\prime \prime} \\
G\left(x, x^{\prime}, x^{\prime \prime}\right)=V\left(x, x^{\prime}\right) V\left(x^{\prime}, x^{\prime \prime}\right) \cos \theta
\end{gathered}
$$

其中, $x, x^{\prime}, x^{\prime \prime}$ 表示场景中的点, $L\left(x, x^{\prime}\right)$ 表示点 $x^{\prime}$ 射向点 $x$ 的辐射亮度, $V\left(x, x^{\prime}\right)$ 表示点 $x$ 和点 $x^{\prime}$ 的可见性, $\Omega / 2$ 表示积 分的范围是点 $x^{\prime}$ 的法向所在的半球面内的所有点 $x^{\prime \prime}, \rho\left(x, x^{\prime}, x^{\prime \prime}\right)$ 表示光线沿点 $x^{\prime \prime}$ 到点 $x^{\prime}$ 的方向入射, 沿点 $x^{\prime}$ 到点 $x$ 的方向出射时的 BRDF 的值, $\theta$ 表示从点 $x^{\prime}$ 到点 $x^{\prime \prime}$ 的方向和点 $x^{\prime}$ 的法向的夹角.

$R_{1}$

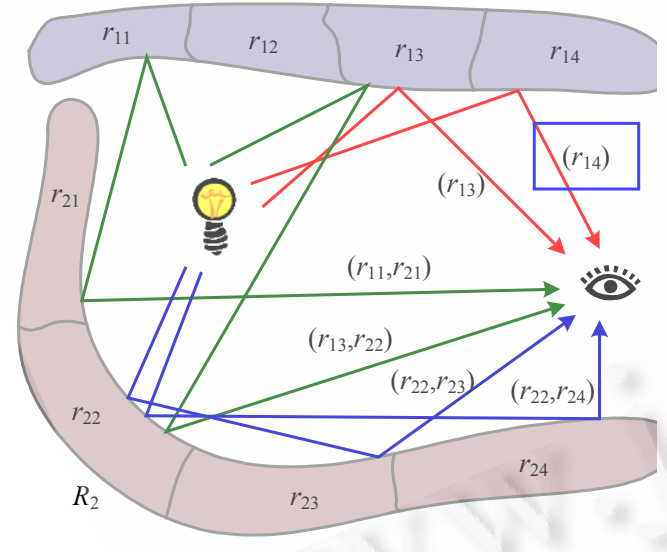

Fig.2 The light transport paths. $\left(r_{13}\right),\left(r_{14}\right)$ :

Direct lighting reflected by $\left(R_{1}\right)$.

$\left(r_{11}, r_{21}\right),\left(r_{13}, r_{22}\right)$ : Indirect lighting reflected by

$\left(R_{1}, R_{2}\right) .\left(r_{22}, r_{23}\right),\left(r_{22}, r_{24}\right)$ : Indirect lighting reflected by $\left(R_{2}, R_{2}\right)$

图 2 光的传播路径. $\left(r_{13}\right),\left(r_{14}\right)$ : 经过 $\left(R_{1}\right)$ 的直接 光照. $\left(r_{11}, r_{21}\right),\left(r_{13}, r_{22}\right)$ : 经过 $\left(R_{1}, R_{2}\right)$ 的间接光照.

$\left(r_{22}, r_{23}\right),\left(r_{22}, r_{24}\right)$ : 经过 $\left(R_{2}, R_{2}\right)$ 的间接光照

在式(1)的描述中,出射的辐射亮度可能经过场景不确定 次数的反射; 为了在出射的辐射亮度和物体材质之间建立线

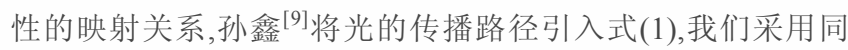
样的方法,所不同的是,我们不需要每个区域内部的材质保持 一致,因此我们将每一个区域 $R$ 分为 $n_{R}$ 个内部材质一致的子区 域来近似模拟:

$$
\begin{gathered}
L\left(x, x^{\prime}\right)=\sum_{n=0}^{\infty} L_{n}\left(x, x^{\prime}\right) \\
L_{n+1}\left(x, x^{\prime}\right)=\sum_{P_{n}} \sum_{R=1}^{N_{r}} \sum_{r=1}^{n_{R}} \tilde{L}\left(x, x^{\prime}, P_{n}\left(R, r, \rho_{R r}\right)\right)
\end{gathered}
$$

$N_{r}$ 表示场景被划分的区域数量, $\left(R, r, \rho_{R r}\right)$ 表示一个区域、 子区域及其BRDF, $\rho_{R r}$ 表示区域 $R$ 的子区域 $r$ 的BRDF. 如图 2 所 示,场景中共有两个空间动态可变材质区域, 每个又被划分为 4 个子区域,图中标出的 6 条光路,如图题所示,分为 3 组,任意 一组在孙金嗝算法中被同样的区域反射,因此属于同一个光 路,一起计算; 但是为了模拟空间动态材质,本文中必须将不同 子区域反射的光路独立计算. 其 $P_{n}$ 表示从光源出射的光 线经过 $n$ 次反射的过程: $P_{n}=\left(R_{1}, r_{1}, \rho_{R_{1^{\prime}}{ }_{1}}\right)\left(R_{2}, r_{2}, \rho_{R_{2} r_{2}}\right) \ldots$ $\left(R_{n}, r_{n}, \rho_{R_{n} r_{n}}\right) \cdot L_{n}\left(x, x^{\prime}\right)$ 表示点 $x^{\prime}$ 射向点 $x$ 的辐射亮度,并且光在到 达点 $x^{\prime}$ 之前经过了 $n$ 次反射, 但对反射路径没有约 束. $\tilde{L}\left(x, x^{\prime}, P_{n}\right)$ 表示点 $x^{\prime}$ 射向点 $x$ 的辐射亮度, 而且光在到达点 $x^{\prime}$ 之前按 $P_{n}$ 所描述的路径经过了 $n$ 次反射. 显然, $\tilde{L}\left(x, x^{\prime}, P_{n}\right)$ 和 $P_{n}$ 包含的材质的乘积线性相关. 我们采用与孙金致 ${ }^{[9]}$ 相 同的方法得到BRDF空间的一组基,将每个子区域的BRDF都表示成这组基的线性组合: 


$$
\rho_{R r}\left(x, x^{\prime}, x^{\prime \prime}\right)=\sum_{i=1}^{N_{b}} c_{R_{i}} \tilde{\rho}_{i}\left(x, x^{\prime}, x^{\prime \prime}\right)
$$

$\tilde{\rho}_{i}$ 表示第 $i$ 个 BRDF 基, $c_{R_{i}}$ 表示 $\rho_{R r}$ 对应于第 $i$ 个基的系数, $N_{b}$ 是基的数量.那么式(2)可以得到:

$$
L_{n+1}\left(x, x^{\prime}\right)=\sum_{P_{n}} \sum_{R=1}^{N_{r}} \sum_{r=1}^{n_{R}} \sum_{i=1}^{N_{b}} c_{R_{i}} \tilde{L}\left(x, x^{\prime}, P_{n}\left(R, r, \tilde{\rho}_{i}\right)\right)
$$

式(4)递归可得:

$$
L_{n}\left(x, x^{\prime}\right)=\sum_{\left\{R_{i}\right\}=1}^{N_{r}} \sum_{\left\{r_{i}\right\}=1}^{n_{R_{i}}} \sum_{\left\{\rho_{i}\right\}=1}^{N_{b}}\left(\tilde{L}\left(x, x^{\prime}, P_{n}\right) \prod_{i=1}^{n} c_{R_{i} r_{i} \rho_{i}}\right) \text { subject to } \rho_{R_{i} r_{i}}=\tilde{\rho}_{\rho_{i}}
$$

我们引入如下表示形式:

$$
\tilde{L}^{\prime}\left(x, x^{\prime}, P_{n}, \rho\right)=\tilde{L}\left(x, x^{\prime}, P_{n}\right) \text { if } \rho_{R^{\prime} r^{\prime}}=\rho
$$

其中, $\rho_{R^{\prime} r^{\prime}}$ 表示反射点 $x^{\prime}$ 所在的子区域的 BRDF.将式(6)代入式(5)可表示为

$$
L_{n}\left(x, x^{\prime}\right)=\sum_{\rho^{\prime}=1}^{N_{b}} c_{R^{\prime} r^{\prime} \rho^{\prime}} \sum_{\left\{R_{i}\right\}=1}^{N_{r}} \sum_{\left\{r_{i}\right\}=1}^{n_{R_{i}}} \sum_{\left\{\rho_{i}\right\}=1}^{N_{b}}\left(\tilde{L}^{\prime}\left(x, x^{\prime}, P_{n}, \rho^{\prime}\right) \prod_{i=1}^{n} c_{R_{i} r_{i} \rho_{i}}\right) \text { subject to } \rho_{R_{i} r_{i}}=\tilde{\rho}_{\rho_{i}}
$$

在本文中,我们的实现只处理两次反射的效果,因此式(2)、式(7)可简化得到:

$$
\begin{aligned}
& \tilde{L}\left(x_{e}, x^{\prime}\right)=L_{0}\left(x_{e}, x^{\prime}\right)+L_{1}\left(x_{e}, x^{\prime}\right) \\
& L_{0}\left(x_{e}, x^{\prime}\right)=\sum_{\rho^{\prime}=1}^{N_{b}} c_{R^{\prime} r^{\prime} \rho^{\prime}} \tilde{L}^{\prime}\left(x_{e}, x^{\prime}, P_{0}, \rho^{\prime}\right) \\
& L_{1}\left(x_{e}, x^{\prime}\right)=\sum_{\rho^{\prime}=1}^{N_{b}} c_{R^{\prime} r^{\prime} \rho^{\prime}} \sum_{R_{1}=1}^{N_{r^{\prime}}} \sum_{r_{1}=1}^{n_{R_{1}}=1} \sum_{\rho_{1}=1}^{N_{b}}\left(\tilde{L}^{\prime}\left(x_{e}, x^{\prime}, P_{1}, \rho^{\prime}\right) c_{R_{1^{\prime}} \rho_{1}}\right)
\end{aligned}
$$

其中, $x_{e}$ 表示视点位置. $\tilde{L}\left(x_{e}, x^{\prime}\right)$ 就是我们需要的最终结果.

为了减少预计算的数据量,我们不考虑BRDF非漫反射部分的互相影响. 与孙釒㙦 ${ }^{[9]}$ 使用同样的假设 $\tilde{\rho}_{1}$ 表示 漫反射BRDF的基,其他的都是非漫反射BRDF的基,因此在式(8)中,我们进一步假设:

$$
\tilde{L}^{\prime}\left(x_{e}, x^{\prime}, P_{1}, \rho^{\prime}\right)=0 \text { if } \rho^{\prime} \neq \widetilde{\rho}_{1} \text { and } \rho_{R_{1^{\prime}}} \neq \widetilde{\rho}_{1}
$$

类似的假设普遍用于重光照技术中,比如 $\mathrm{Hasan}^{[10]}$ 假设所有的反射都是漫反射; 与 $\mathrm{Hasan}^{[10]}$ 不同的是,我们 的假设中还保留了从非漫反射到漫反射的间接光照,所以我们能够保留焦散等高频的间接光照的绘制效果.

在以上的数学描述中,如果我们把每个子区域都看作一个独立的区域,那么实际上可以等价于孙釒政 ${ }^{[9]}$ 的方 法.但是, 孙釒金 ${ }^{[9]}$ 的方法中并没有对 $\widetilde{L}^{\prime}$ 进行压缩. 我们可以看到, 由于引入了子区域,因此 $\widetilde{L}^{\prime}$ 数量急剧增加, 不进行 合理的压缩是无法进行绘制的. 我们将子区域作为独立于区域的变量引入, 是因为对于不同的区域所对应的 $\widetilde{L}^{\prime}$ 分别进行压缩,而不是将所有的 $\widetilde{L}^{\prime}$ 一起压缩. 如图 2 所示,分为一组的光路是被同样的区域反射得到,其所包含 的不同子区域对应的光路的预计算的结果被作为一组进行压缩.我们采用这样的压缩策略主要有 4 个原因: 一 般情况下区域的划分有实际意义,比如室内的地板和墙壁,但是子区域没有,因此我们可以认为,一个区域内部 的子区域相关性更强,更利于压缩; 不同区域之间的材质差异明显,同一区域内部的材质许多时候体现出相似的 特点,如果在一起压缩,则可能会导致两种差异巨大的材质互相污染串色; 将数据分为多个组, 各自压缩到数量 较少的基, 与所有数据压缩到一组数量较大的基相比,在绘制效果相当的情况下,前者的绘制性能更优;对于不 同的区域,可以根据实际视觉效果的敏感程度采用不同的压缩率.

\section{3 预计算及数据压缩}

场景区域的划分由用户指定.对于每一个区域,我们先赋予形变尽量小的纹理坐标,并在纹理坐标空间中均 匀划分,然后对应到原始的几何上形成子区域.我们采用 DirectX 的 UVAtlas 工具赋予纹理坐标,得到良好的效 果.实际上,子区域的划分结果对于效果而言是十分重要的,如果划分得过于粗粘,就会丢失过多的材质变化的 细节,划分得过于细致,就会给绘制带来巨大的代价.显而易见,子区域划分得越细密,预计算结果的压缩空间就 
越大. 因此我们采用高密划分、高度压缩的方法, 首先将场景划分成尽量小的子区域, 以获取尽量多的材质的空 间动态效果,然后在压缩时可以大量抛弃不敏感或者暗淡的部分, 以平衡绘制效果和绘制性能.

我们采用与孙金致 ${ }^{[9]}$ 一样的方法可以得到所有的 $\widetilde{L}^{\prime}$. 所有可见的点 $x^{\prime}$ 就是对应绘制结果中的每个像素, 因此 可以将 $\widetilde{L}^{\prime}$ 存储成高动态范围 (high dynamic range 简称 HDR) 图像: 将 $\tilde{L}^{\prime}\left(x_{e}, x^{\prime}, P_{0}, \rho^{\prime}\right)$ 存储成 $I\left(P_{0}, \rho^{\prime}\right)$, 将 $\tilde{L}^{\prime}\left(x_{e}, x^{\prime}, P_{1}, \rho^{\prime}\right)$ 存储成 $I\left(P_{1}, \rho^{\prime}\right)$. 此外, 由于最终绘制的要求, 还需要同样分辨率的场景材质区域分布映射图像, 不 但记录每个像素所在的区域和子区域,而且记录每个像素的纹理坐标.

得到 $I$ 之后, 需要对其进行压缩. 我们保留所有的 $I\left(P_{0}, \rho^{\prime}\right)$, 只对 $I\left(P_{1}, \rho^{\prime}\right)$ 进行压缩. 这是因为 $I\left(P_{0}, \rho^{\prime}\right)$ 用于直接 光照的结果, 体现了大量的高频细节, 压缩很容易影响绘制效果, 而且 $I\left(P_{0}, \rho^{\prime}\right)$ 的数据量并不大, 与区域和子区域 的数量无关; 相反地, $I\left(P_{1}, \rho^{\prime}\right)$ 用于间接光照, 较为低频, 而且数据量巨大, 需要进行压缩. 根据以上理由, 我们对不 同区域的 $I\left(P_{1}, \rho^{\prime}\right)$ 分别进行压缩. 事实上, $I\left(P_{1}, \rho^{\prime}\right)$ 有很明显的稀疏性和相关性: 稀疏性是因为我们将子区域划分 得较小,互相可见的子区域数量较少,而且许多子区域由于和光源可见程度低,或者距离可见的其他子区域距离 较远, 对全局光照明的贡献就很弱了; 相关性是因为辐射强度经过的反射次数越多就越低频 ${ }^{[30]}$, 同一区域的子区 域互相之间材质较为接近, 空间位置相似.这种场景内光照传输的稀疏性和相关性被广泛应用于全局光照明的 算法中 ${ }^{[10,30]}$. 一个区域 $R_{1}$ 对应的 $I\left(P_{1}, \rho^{\prime}\right)$ 需要进一步根据 $\rho^{\prime}$ 的不同划分为不同的组分别压缩, 这是因为不同的 $\rho^{\prime}$ 其图像的亮度有很大的区别, 如果 $\rho^{\prime}$ 对应于BRDF的基的高频部分, 图像的亮度都很弱, 但是对于绘制效果而 言却十分敏感而重要,如果放在一起压缩,会丢失这些高频的绘制效果.

那么一个 $\left(R_{1}, \tilde{\rho}\right)$ 对应的 $I\left(P_{1}, \rho^{\prime}\right)$ 可以看作是一个三维数据:3 个维度包括 $r_{1}$ 和图像的宽度和高度. 我们要对 这 3 个维度都进行压缩: 对这些 $I\left(P_{1}, \rho^{\prime}\right)$ 图像在维度 $r_{1}$ 使用PCA进行压缩,得到一组基;然后对这组基中的每一个 基图像用Harr小波进行压缩.我们采用PCA压缩所有的 $I\left(P_{1}, \rho^{\prime}\right)$ 而不是采用Harr小波有 3 个原因:在较高的压缩 率下,Harr会引入高频误差, 严重影响绘制效果, 而PCA引入的误差较为低频, 视觉效果不敏感; 在图像空间的两 个维度上由于需要保持高频细节效果,所以压缩率受到限制,因此在维度 $r_{1}$ 上压缩时需要采用较高的压缩率; 在 维度 $r_{1}$ 上进行PCA压缩是一种非常有效的抑制图像噪点的方法 ${ }^{[31]}$.

在进行Harr小波压缩之前, 我们需要对每个基图像进行中值滤波.在进行预计算时,我们会引入路径跟踪固 有的噪点, 而噪点对Harr小波压缩的影响非常大. 这些噪点相对于最终的绘制结果而言是不明显的, 但是当它们 分别作为预计算的每个数据存储时,却明显许多,基于和孙釒政同样的理由,我们不能对路径跟踪进行重要性采 样 ${ }^{[32]}$, 因此通过提高采样光线数量的方法减弱噪点的代价就会非常大. 对图像进行PCA压缩本身就具有降噪的 作用 ${ }^{[31]}$, 但是效果并不足以保证Harr小波压缩时保留主要信息而不是保留大量的噪点信息.中值滤波是一个行 之有效的降噪方法 ${ }^{[33]}$, 需要注意的是, 在中值滤波过程中, 不同的区域互相对比明显, 因此不能将不同区域的像 素放在一起滤波, 否则会造成明显的串色而影响绘制效果; 但是同一区域的不同子区域之间却必须一起滤波, 否 则绘制结果中子区域的交界处会出现明显的不连续现象. 这样经过滤波的基图像进行Harr小波压缩时能够在 较高的压缩率下依然保持良好的绘制效果.

\section{4 材质编辑和绘制}

我们采用Blinn-Phong 的BRDF模型 ${ }^{[3]}$ :

$$
\rho_{B P}=\frac{K_{d}}{\pi}+K_{s} \frac{s+2}{2 \pi} \cos ^{s} \delta
$$

其中, $\delta$ 表示入射方向和出射方向的中间方向和反射点法向的夹角, $s$ 表示物体表面的粗粘程度, 为正实数, $K_{d}$ 表示 漫反射的反射率, $K_{s}$ 表示非漫反射的反射率.

如上文所述,对于每个空间动态可变材质区域,我们都赋予了纹理坐标. 因此,可以用纹理的方式给场景进 行材质编辑. 我们对每个空间动态可变材质区域赋予 3 张三通道的HDR(high dynamic range)图像作为材质纹 理,分别表示 $\mathrm{RGB}$ 三通道的 $K_{d}, K_{s}$ 和 $s$, 该区域每个点的材质都可以根据其纹理坐标在这 3 张图像上取得相应的 值.通过编辑修改相应的纹理,我们就能完成材质编辑. 
根据式(8), 使用 $I\left(P_{0}, \rho^{\prime}\right)$ 和压缩后的 $I\left(P_{1}, \rho^{\prime}\right)$ 进行绘制.对于点 $x^{\prime}$ 的材质 $\rho^{\prime}$, 我们根据预计算时得到场景材质 区域分布映射图像中的纹理坐标, 索引材质纹理得到该点准确的BRDF参数. 对于 $P_{1}$ 中的 $\rho_{R_{1^{\prime}}}$, 我们通过对材质 纹理的降采样得到,降采样的分辨率取决于在纹理坐标空间划分子区域的分辨率.

由于参与绘制的数据量太大, 难以放入显存, 因此我们采用 CPU 进行绘制, 而没有采用 GPU 进行加 速. $I\left(P_{0}, \rho^{\prime}\right)$ 由于不需要压缩, 因此绘制方法与文献[9]相似,所不同的是, 我们采用 CPU 绘制, 并且对每一个像素 都对应不同的材质系数,而不像文献[9]中同一区域的像素具有相同的系数. $I\left(P_{1}, \rho^{\prime}\right)$ 以压缩之后的小波系数的 形式存在,因此我们在计算组合 $I\left(P_{1}, \rho^{\prime}\right)$ 时也是采用在小波空间中计算, 并将最后得到的小波系数投影到图像空 间, 并与 $I\left(P_{0}, \rho^{\prime}\right)$ 的结果相加, 就得到了最后的绘制结果. 绘制性能的主要代价在于组合 $I\left(P_{1}, \rho^{\prime}\right)$, 由于 $I\left(P_{1}, \rho^{\prime}\right)$ 数 量较大, 因此有很高的并行性, 可采用多线程进行加速. 我们将 $I\left(P_{1}, \rho^{\prime}\right)$ 分为多组, 每组分别由一个线程进行组合, 最后再将所有线程的结果相加, 这样能有效提高绘制性能. $I\left(P_{0}, \rho^{\prime}\right)$ 相关的数据量并不大, 因此可以在 GPU 中实 现,但是由于绘制的主要代价在 $I\left(P_{1}, \rho^{\prime}\right)$, 相比之下, GPU 对于 $I\left(P_{0}, \rho^{\prime}\right)$ 的绘制的加速效果可以忽略不计, 因此我 们没有采用这样的方法,而是全部采用 CPU 进行绘制.

\section{5 实验结果}

我们用 VC++2005 和DirectX9 在Windows XP下实现以上算法,运行环境是dual $3.20 \mathrm{GHz}$ Xeon CPU,4.0 Gbytes RAM.BRDF的采样分辨率和孙釒踇 ${ }^{[9}$ 的相同, $s \in[1,200]$,对于直接光照, 我们采用 $N_{b}=6$ 个基, 间接光照, 我 们采用 $N_{b}=4$ 个基. 实验的场景包含一个空间动态材质可变的区域, 子区域数量为 $32 \times 32=1024$,一个空间静态 材质可变的区域,PCA压缩率为 $12.5 \%$,Harr小波压缩保留 $13.3 \%$ 的系数, 绘制分辨率为 $512 \times 512$, 修改材质时的绘 制速度为 $2.41 \mathrm{fps}$. 预计算得到的数据量为 21.0 Gbytes, 压缩后为 781 Mbytes.

对于直接光照的 BRDF 的基函数的个数选择, 我们采用文献[9]中的结论, 文献[9]已经给出的数值误差和绘 制结果与参考图像的比较证明这个结论的有效性. 对于两次反射的间接光照, 我们采用 4 个基函数, 这是因为反 射次数越多, 光照效果就越低频,因此我们可以采用较少的基函数. 采样 BRDF 的 4 个基函数的重建的 L2 误差为 $5.53 \%$. 子区域的数量取决于我们的系统能够承受多大的数据量和运算代价, 因为我们采用高密划分、高度压缩 的策略,并没有对于子区域的划分作特别处理, 用户可以在性能和效果的权衡下选择自己的参数设置. 通过下面 的实验结果和参考图象的对比可以看出,我们的参数设置能够在有限的预计算时间内生成良好的视觉效果.

表 1 中记录了所有实验结果相关的场景的规模和预计算的参数和性能.

Table 1 Performance of precomputation on exhibited examples

表 1 所示实验的预计算的性能

\begin{tabular}{ccccccc}
\hline & Number & Number of & \multicolumn{2}{c}{ Number of light sampling points } & \multicolumn{2}{c}{ Time (hour) } \\
\cline { 3 - 6 } & of faces & sampling rays & Direct lighting & Indirect lighting & Precomputation & Compression \\
\hline Cubes & 38 & 16384 & 800 & 2 & 8.0 & 8.2 \\
Bunny & $23.2 \mathrm{k}$ & 6400 & 800 & 2 & 7.8 & 8.4 \\
Tube & $1.2 \mathrm{k}$ & 10000 & 1 & 1 & 2.2 & 7.3 \\
\hline
\end{tabular}

在后文给出的数据中,每张绘制效果下面的 3 张纹理,从左到右分别赋给代表空间动态可变材质区域的 $K_{d}$, $K_{s}$ 和 $s$.

在图 3 中左右墙面、地板和 cubes 后面的墙面构成一个空间动态可变材质区域.Cubes 被赋予高光材质,我 们可以看到周围墙面在 cube 上明显的渗色效果. 其中图 3(b)、图 3(c)墙面的材质有明显的区域和形态, 可以在 cubes 上看到较为清晰的投影.

在图 4 中,Bunny 是一个空间动态可变材质区域.四周的平面是一个空间静态可变材质区域,我们可以看到 Bunny 和四周墙面的明显的互相反射的亮度. 其中图 4(a)、图 4(b)四周平面被赋予接近漫反射的材质; 图 4(c)四 周平面被赋予高光材质, 受到 Bunny 的反射效果也就更加明显. 此外, 我们可以看到图 4(a)中, Bunny 上背部和头 部区域的相互反射的效果.

在图 5 中 tube 是一个空间动态可变材质区域,平面是一个空间静态可变材质区域.我们可以看到,tube 上高 
频的材质分布能够在平面上投影出清晰的投影. 其中图 5(b)、图 5(c)圆环具有高光的材质,因此在平面上有明显 的焦散.图 5(a)、图 5(c)平面被赋予了高光材质,因此图 5(a)圆环在平面上有清晰的倒影, 图 5(c)的焦散比图 5(b) 更为明显和强烈.

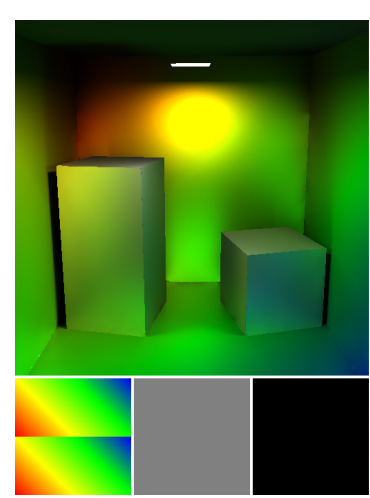

(a)

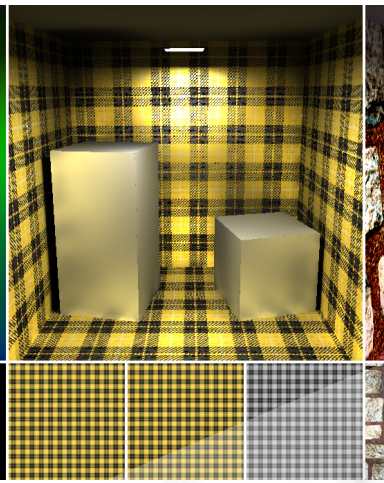

(b)

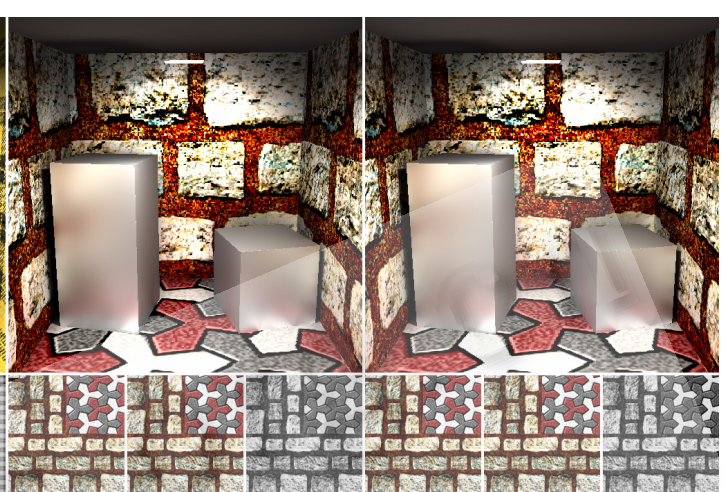

(c)

(d)

Fig.3 Cubes. (d) is the ground truth of (c), L2 error is 7.518e-004

图 3 Cubes. (d) 是 $(\mathrm{c})$ 的参考图像, $\mathrm{L} 2$ 误差为 $7.518 \mathrm{e}-004$

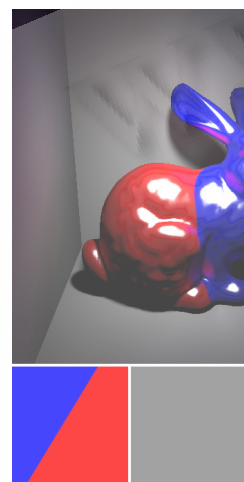

(a)

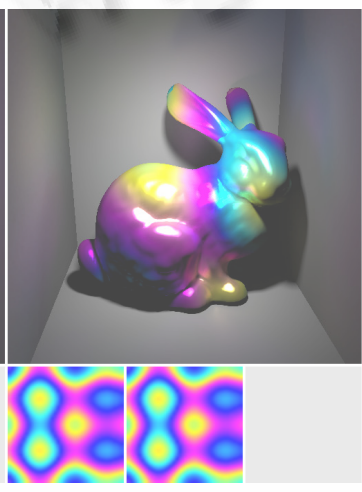

(b)

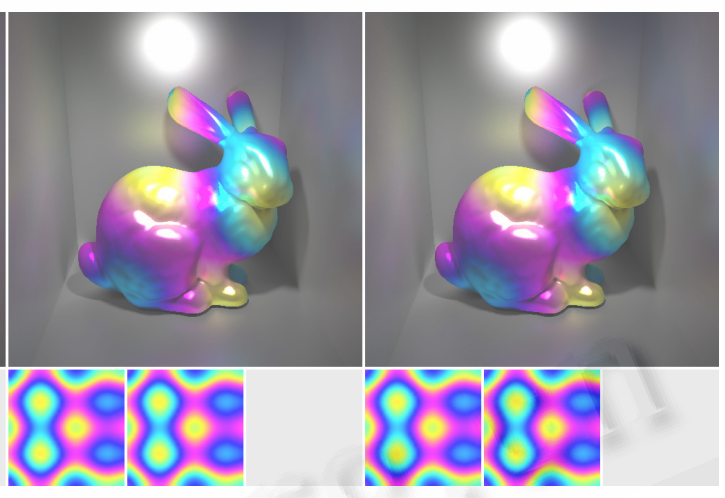

(c)

(d)

Fig.4 Bunny. (d) is the ground truth of (c), L2 error is $2.8787 \mathrm{e}-005$

图 4 Bunny. (d) 是(c)的参考图像, L2 误差为 $2.8787 \mathrm{e}-005$

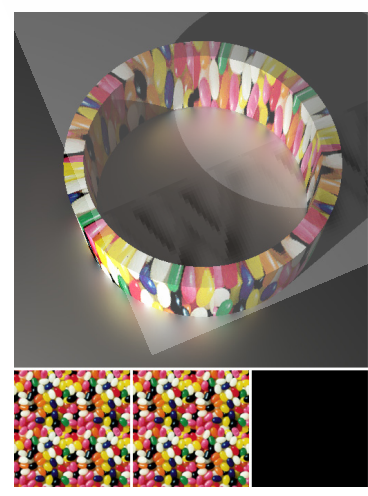

(a)
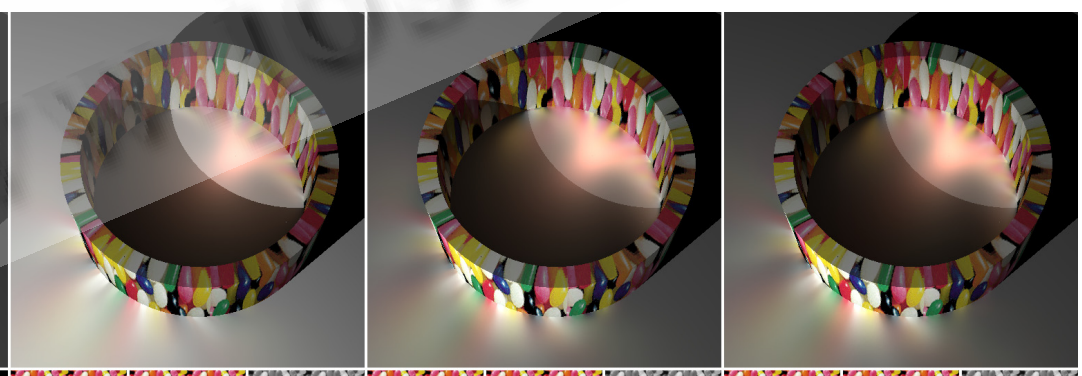

(a) 178

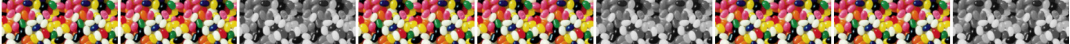

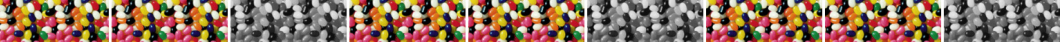

(b) (c)

(d)

Fig.5 Tube. (d) is the ground truth of (c), L2 error is $3.4280 \mathrm{e}-004$

图 5 Tube. (d) 是 $(\mathrm{c})$ 的参考图像, L2 误差为 $3.4280 \mathrm{e}-004$ 


\section{6 结束语}

\section{1 结 论}

本文的方法实现了空间动态可变材质的交互式全局光照明绘制. 我们将空间动态可变材质区域划分为多 个空间静态可变材质的子区域.虽然会产生巨大的预计算的数据量,但是由于子区域之间的辐射亮度传输有很 高的稀疏性和相关性,因此我们可以将与计算得到的数据压缩到可以接受的范围来达到交互式的绘制性能.

\section{2 局限性讨论}

本文的方法具有十分现实的应用背景,比如能够有效地提高游戏、电影等的三维场景建模时材质编辑的效 率.空间动态材质及其全局光照明对于场景的视觉效果是至关重要而且十分敏感的,但是同时具有极高的时间 和空间的复杂度,在现有的硬件条件和技术水平下是难以在不作任何约束或假定的一般性情况下解决这个问 题的. 因此, 本文的方法出于数据量和性能的考虑, 对绘制的条件作了一些相应的限制, 主要有以下 3 点:(1) 场景 几何结构、光照条件和视点方向固定不变;(2) 只考虑一次反射形成的直接光照和两次反射形成的间接光照的 效果,不考虑两次以上的反射形成的间接光照效果;(3) 不考虑 BRDF 的高光部分的相互反射效果.

后两个对间接光照的假设会影响全局光照明的绘制效果,但是如果不作这样的假设,除了会带来巨大的数 据量, 极大地降低预计算和绘制的性能以外, 同时能够得到的绘制效果的提升也非常有限, 因为在许多现实场景 和材质条件下, 两次反射已经能够描述主要的光照效果, 并且在它们的承托下, 其他被忽略的光照效果并不会带 来明显的视觉差异. 因此这两个假设广泛用于全局光照明的算法, 如文献[10]只考虑漫反射的间接光照效果, 文 献[2]只处理直接光照的绘制效果. 而在我们的实验数据中, 算法的绘制结果和参考图像十分接近, 充分说明这两 个假设并不会影响我们的算法在许多场合的应用价值. 只有当场景中有高光材质之间强烈的反射时,比如多次 的镜面反射和折射,这两个假设才有可能造成绘制结果和真实效果的较大差异, 这也是我们方法的局限性. 如果 需要解决对这类场景的应用问题,则需要进一步的研究工作,采用更具针对性的方法.

而对我们算法的实际应用局限最大的是对光源和视点固定不变的假设,因为这要求在场景设计和编辑的 时候就要求先确定光照和视点方向, 然后再进行材质编辑, 而不能同时进行. 但对于几何结构不变的假设却不会 过于限制我们方法的应用, 因为在实际应用中,比如室内装潢、电影及游戏的场景设计中,很少是通过改变几何 结构来调整光照效果的, 事实上, 都是先确定几何结构再进行光照效果的设计. 目前相关工作中只有文献[8]能在 绘制全局光照明效果的同时改变光照、视点和材质,但却不能处理空间动态可变材质.也就是说,无法承担空间 动态可变材质所带来的巨大的数据量和预计算绘制的运算代价,因此也无法解决本文的问题.

综上所述,虽然我们的算法依赖一些假设和约定,但并不会影响本文算法的有效性和实际应用价值.原因总 结起来有 4 点:

（1）这些假设是出于对数据量和运算性能考虑的合理假设,并为相关工作广泛采用;

(2) 对于大多数应用场景能够提供足够的效果和性能的保证;

(3) 本文首次解决了空间动态可变材质的交互级全局光照明绘制,这是前人工作所不能解决的问题;

(4) 对用户的额外要求(先确定光照和视点然后进行材质编辑) 并不严苛, 虽然并不十分完美, 但是相对于 非交互级的编辑和预览绘制效果,已经能够带来极大的便利.

\section{3 后续工作}

更有效地划分子区域,可在不增加预计算数据量的同时,抓取更多的全局光照明的绘制效果.进一步压缩预 计算数据量并提高绘制速度,对不同反射次数的间接光照采用不同的预计算和压缩的方法,可以扩展到实现更 多次的反射效果.如果可以进一步扩展以支持动态光源和可变视点,则能对实际应用带来更多的便利.

\section{References:}

[1] Kajiya JT. The rendering equation. In: Evans DC, ed. Proc. of the SIGGRAPH'86. New York: ACM Press, 1986. 143-150. 
[2] Ben-Artzi A, Overbeck R, Ramamoorthi R. Real-Time BRDF editing in complex lighting. In: Hart JC, ed. Proc. of the SIGGRAPH 2006. New York: ACM Press, 2006. 945-954.

[3] Blinn JF. Models of light reflection for computer synthesized pictures. In: George J. ed. Proc. of the SIGGRAPH'77. New York: ACM Press, 1977. 192-198.

[4] Appel A. Some techniques for shading machine renderings of solids. In: Proc. of the Spring Joint Computer Conf. Arlington: AFIPS Press, 1968. 37-45.

[5] Jensen HK. Realistic Image Synthesis Using Photon Mapping. Natick: A K Peters, Ltd., 2001. 1-181.

[6] Sloan PP, Kautz J, Snyder J. Precomputed radiance transfer for real-time rendering in dynamic, low-frequency lighting environments. In: Fiume E, ed. Proc. of the SIGGRAPH 2002. New York: ACM Press, 2002. 527-536.

[7] Zhou K, Hu Y, Lin S, Guo B, Shum H. Precomputed shadow fields for dynamic scenes. In: Hart JC, ed. Proc. of the SIGGRAPH 2005. ACM Press, 2005. 1196-1201.

[8] Sun X, Zhou K, Chen Y, Lin S, Shi J, Guo B. Interactive relighting with dynamic BRDFs. In: Hart JC, ed. Proc. of the SIGGRAPH 2007. New York: ACM Press, 2008. http://portal.acm.org/citation.cfm?id=1276377.1276411\&coll=Portal\&dl=GUIDE\&CFID= 73850906\&CFTOKEN $=30075946$

[9] Sun X, Zhou K, Shi JY. Real-Time global illumination rendering with dynamic materials. Journal of Software, 2008,19(4): 1004-1015 (in Chinese with English abstract). http://www.jos.org.cn/1000-9825/19/1004.htm

[10] Hasan M, Pellacini F, Bala K. Direct-to-Indirect transfer for cinematic relighting. In: Hart JC, ed. Proc. of the SIGGRAPH 2006. New York: ACM Press, 2006. 1089-1097.

[11] Goral CM, Torrance KE, Greenberg DP, Battaile B. Modeling the interaction of light between diffuse surfaces. In: Christiansen H, ed. Proc. of the SIGGRAPH'84. New York: ACM Press, 1984. 213-222.

[12] Dorsey J, Sillion F, Greenberg DP. Design and simulation of opera lighting and projection effects. In: Sederberg TW, ed. Proc. of the SIGGRAPH'91. New York: ACM Press, 1991. 41-50.

[13] Dorsey J, Arvo J, Greenberg DP. Interactive design of complex time-dependent lighting. IEEE Computer Graphics and Applications, 1995,15(2):26-36.

[14] Dobashi Y, Kaneda K, Nakatani H, Yamashita H. A quick rendering method using basis functions for interactive lighting design. Computer Graphics Forum, 1995,14(3):229-240.

[15] Saito T, Takahashi T. Comprehensible rendering of 3-D shapes. In: Baskett F, ed. Proc. of the SIGGRAPH'90. New York: ACM Press, 1990. 197-206.

[16] Gershbein R, Hanrahan PM. A fast relighting engine for interactive cinematic lighting design. In: Akeley K, ed. Proc. of the SIGGRAPH 2000. New York: ACM Press, 2000. 353-358.

[17] Pellacini F, Vidimce K, Lefohn A, Mohr A, Leone M, Warren J. Lpics: A hybrid hardware-accelerated relighting engine for computer cinematography. In: Hart JC, ed. Proc. of the SIGGRAPH 2005. New York: ACM Press, 2005. 464-470.

[18] Sloan PP, Hall J, Hart J, Snyder J. Clustered principal components for precomputed radiance transfer. In: Hodgins J, ed. Proc. of the SIGGRAPH 2003. New York: ACM Press, 2003. 382-391.

[19] Ng R, Ramamoorthi R, Hanrahan P. All-Frequency shadows using non-linear wavelet lighting approximation. In: Hodgins J, ed. Proc. of the SIGGRAPH 2003. New York: ACM Press, 2003. 376-381.

[20] Ng R, Ramamoorthi R, Hanrahan P. Triple product integrals for all-frequency relighting. In: Marks J, ed. Proc. of the SIGGRAPH 2004. New York: ACM Press, 2004. 477-487.

[21] Liu X, Sloan P, Shum HY, Snyder J. All-Frequency precomputed radiance transfer for glossy objects. In: Jensen HW, ed. Proc. of the Eurographics Symp. on Rendering 2004. Aire-la-Ville: Eurographics Association, 2004. 337-344.

[22] Wang R, Tran J, Luebke D. All-Frequency relighting of non-diffuse objects using separable BRDF approximation. In: Jensen HW, ed. Proc. of the Eurographics Symp. on Rendering 2004. Aire-la-Ville: Eurographics Association, 2004. 345-354.

[23] Minnaert M. The reciprocity principle in lunar photometry. Astrophysical Journal, 1941,93(2):403-410.

[24] Cook RL, Torrance KE. A reflectance model for computer graphics. ACM Trans. on Graph., 1982,1(1):7-24.

[25] Phong BT. Illumination for computer generated pictures. Communications of the ACM, 1975,18(6):311-317. 
[26] Ward GJ. Measuring and modeling anisotropic reflection. In: Thomas JJ, ed. Proc. of the SIGGRAPH'92. New York: ACM Press, 1992. 265-272.

[27] Matusik W, Pfister J, Brand M, McMillan L. A data-driven reflectance model. In: Hodgins J, ed. Proc. of the SIGGRAPH 2003. New York: ACM Press, 2003. 759-769.

[28] Ershov S, Kolchin K, Myszkowski K. Rendering pearlescent appearance based on paint-composition modelling. In: Chalmers A, ed. Proc. of the Eurographics 2001. Britain: Blackwell Publishers, 2001. 227-238.

[29] McCool MD, Ang J, Ahmad A. Homomorphic factorizations of BRDFs for high-performance rendering. In: Fiume E, ed. Proc. of the SIGGRAPH 2001. New York: ACM Press, 2001. 227-238.

[30] Durand F, Holzschuch N, Soler C, Chan E, Sillion F. A frequency analysis of light transport. In: Hart JC, ed. Proc. of the SIGGRAPH 2005. ACM Press, 2005. 1115-1126.

[31] Meyer M, Anderson J. Statistical acceleration for animated global illumination. In: Hart JC, ed. Proc. of the SIGGRAPH 2006. New York: ACM Press, 2006. 1075-1080.

[32] Clarberg P, Jarosz W, Akenine-Möller T, Jensen HW. Wavelet importance sampling: Efficiently evaluating products of complex functions. In: Hart JC, ed. Proc. of the SIGGRAPH 2005. ACM Press, 2005. 1166-1175. http://portal.acm.org/citation.cfm?id= $1186822.1073328 \&$ coll $=$ Portal $\& \mathrm{dl}=$ GUIDE\&CFID $=73850906 \&$ CFTOKEN $=30075946$

[33] Lee ME, Redner RA. A note on the use of nonlinear filtering in computer graphics. IEEE Computer Graphics and Applications, $1990,10(3): 23-29$.

\section{附中文参考文献：}

[9] 孙金金,周昆,石教英.可变材质的实时全局光照明绘制.软件学报,2008,19(4):1004-1015. http://www.jos.org.cn/1000-9825/19/ 1004.htm

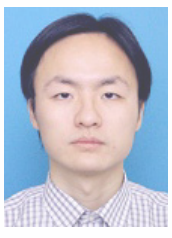

孙唫(1980-), 男, 浙江杭州人,博士生,主 要研究领域为实时绘制.
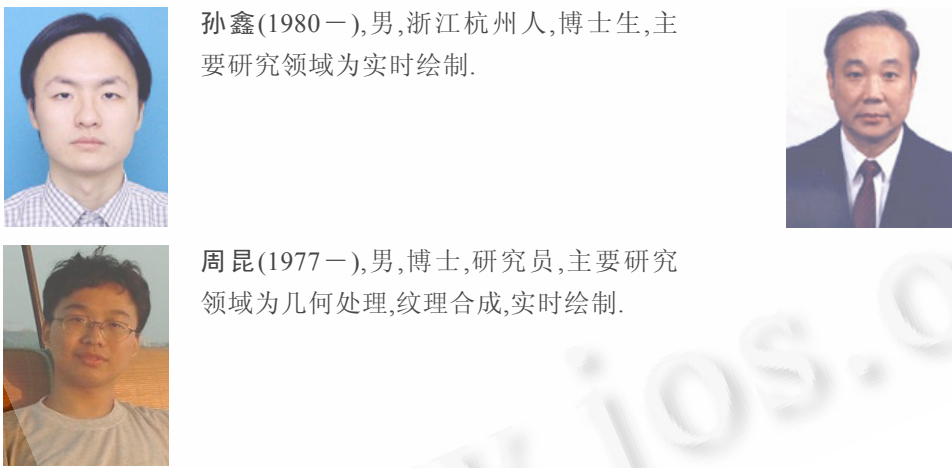

石教英(1937-),男,教授,博士生导师, CCF 高级会员,主要研究领域为分布式计算,虚 拟现实,科学计算可视化,多媒体技术.

周昆(1977-), 男, 博士, 研究员, 主要研究 领域为几何处理,纹理合成, 实时绘制. 